\title{
Catalpol Enhances Neurogenesis And Inhibits Apoptosis Of New Neurons Via BDNF, But Not The BDNF/Trkb Pathway
}

This article was published in the following Dove Press journal:

Drug Design, Development and Therapy

\author{
Hui-Feng Zhu $\mathbb{D}^{1, *}$ \\ Yali Shao $\mathbb{D D}^{1, *}$ \\ Lei Qin ${ }^{1, *}$ \\ Jing-Huan Wang' \\ Shan Feng' \\ Yun-Bin Jiang' \\ Dong Wan $\mathbb{1}^{2}$
}

'Department of Pharmacy, College of Pharmaceutical Sciences and Chinese Medicine, Southwest University, Chongqing 4007I5, People's Republic of China; ${ }^{2}$ Department of Emergency, The First Affiliated Hospital of Chongqing Medical University, Chongqing 400016, People's Republic of China

*These authors contributed equally to this work
Correspondence: Hui-Feng Zhu Department of Pharmacy, College of Pharmaceutical Sciences and Chinese Medicine, Southwest University, Chongqing 4007I5, People's Republic of China Email zhfbswu@swu.edu.cn

Dong Wan

Department of Emergency, The First Affiliated Hospital of Chongqing Medical University, Chongqing 400016, People's

Republic of China

$\mathrm{Tel}+86-23-68251225$

Email wandongcqykdx@126.com
Background: The role of catalpol in brain neurogenesis and newborn neuron survival has not been previously determined in permanent middle cerebral artery occlusion (pMCAO).

Methods: Fifty-four rats were divided into 6 groups: pMCAO (model, $\mathrm{n}=9$ ); sham operation (NS, $\mathrm{n}=9$ ); catalpol treatment ( $5 \mathrm{mg} / \mathrm{kg}$ and $10 \mathrm{mg} / \mathrm{kg}$ subgroups, $\mathrm{n}=9$ each); K252a ( $=9$ ); and $\mathrm{K} 252 \mathrm{a}+$ catalpol $5 \mathrm{mg} / \mathrm{kg}(\mathrm{n}=9)$ with stroke. The effects of catalpol on behavior, neurogenesis surrounding the infarction ipsilateral to pMCAO, and the expression of brainderived neurotrophic factor (BDNF) and its receptor (TrkB) were evaluated. Vehicle or, K252a (i.p.), an inhibitor of TrkB phosphorylase.

Results: Repeated administration of catalpol reduced neurological deficits and significantly improved neurogenesis. Catalpol increased the number of newborn immature neurons, as determined by $\mathrm{BrdU}^{+}-\mathrm{Nestin}^{+}$and $\mathrm{BrdU}^{+}-\mathrm{Tuj}_{-1}^{+}$staining, and downregulated cleaved caspase 3 in Tuj $-1^{+}$cells at day 7 following stroke. Moreover, catalpol increased the protein expression of Tuj-1, MAP2, and the Bcl-2/Bax ratio, as determined using Western blot. Catalpol also significantly increased brain levels of BDNF, but not TrkB, resulting in enhanced survival of newborn neurons via inhibition of apoptosis.

Conclusion: Catalpol may contribute to neurogenesis in infarcted brain regions and help promote the survival of newborn neurons by activating BDNF, but not BDNF/TrkB signaling.

Keywords: catalpol, neurogenesis, permanent middle cerebral artery occlusion, pMCAO, brain derived neurotrophic factor, BDNF, neurological function

\section{Introduction}

Endogenous neural stem cells are potential therapeutic targets to produce new neurons after stroke and have been shown to migrate to the borders of ischemic lesions. ${ }^{1,2}$ However, apoptosis of newborn neurons may limit these endogenous repair mechanisms following stroke. ${ }^{1-3,5}$ Recent data showed that an enriched vascular environment is involved in proliferation, differentiation, migration, and survival of neural progenitor cells following stroke. ${ }^{3-5}$ In addition, secretion of trophic factors ${ }^{4,6}$ including brain-derived neurotrophic factor (BDNF), ${ }^{4,6}$ erythropoietin (EPO), ${ }^{7}$ and vascular endothelial growth factor $(\mathrm{VEGF})^{8}$ may provide trophic support for the survival of newborn neurons, allowing for the construction of neuronal circuits in situ. ${ }^{5}$ Therefore, pharmacological interventions aimed at restoring neurological function following brain injuries via increased angiogenesis and neurogenesis could be potential therapies for stroke. 
Catalpol, purified from Rehmannia glutinosa Libosch, has been shown to upregulate VEGF, EPO, and BDNF to promote angiogenesis in infarcted regions of the brain via the JAK2/STAT3 pathway, and to ameliorate brain capillary endothelial cell edema. ${ }^{9-11}$ However, the effects of catalpol on neurogenesis have not yet been characterized. A previous study showed that treatment with catalpol and puerarin (C-P) promoted angiogenesis and neurogenesis. ${ }^{12}$ We also showed that C-P promoted angiogenesis, ${ }^{9,11}$ but the effects of catalpol alone have not been characterized.

In this study, we investigated the pro-neurogenic/survival effects of catalpol and the relationship of catalpol's anti-apoptotic effects with BDNF and its receptor TrkB, including neurobehavioral function recovery assessment.

\section{Materials And Methods}

\section{Animals And pMCAO Model}

All experiments were performed in accordance with the Basel Declaration and China's Guidelines for Care and Use of Laboratory Animals; the experimental protocol was approved by the Experimental Animal Ethics Committee of College of Pharmaceutical Sciences \& Chinese Medicine, Southwest University. Healthy male Sprague-Dawley (SD) rats (3 months old, 220-250 g) were obtained from the Experimental Animal Center at the Southwest University of Medicine, China, and housed under natural illumination with ad libitum access to food and water. Stroke was induced by electrocoagulation of the right middle cerebral artery (pMCAO) as previously described. ${ }^{13,14}$ The rats were prescreened to select those that met the neurological criteria according to Bederson. ${ }^{6,14}$

\section{Groups And Drug Treatment}

The animals were randomly divided into 6 groups, with 9 rats in each group: $\mathrm{p}-\mathrm{MCAO}$ (model, $\mathrm{n}=9$ ); sham-operated; $5 \mathrm{mg} / \mathrm{kg}$ catalpol; $10 \mathrm{mg} / \mathrm{kg}$ catalpol; K252a; and K252a with $5 \mathrm{mg} / \mathrm{kg}$ catalpol. Catalpol (>98\% purity, National Institute for the Control of Pharmaceutical and Biological Products, Peking, China) was dissolved in physiological saline and administered $6 \mathrm{~h}$ after pMCAO, then administered daily for 7 days at doses of 5 or $10 \mathrm{mg} / \mathrm{kg}$ body weight. The injection concentrations were 0.5 and $1 \mathrm{mg} /$ $\mathrm{mL}$. The sham-operated group and the vehicle group received physiological saline $(100 \mathrm{~g} / \mathrm{mL}$, i.p.). K252a $(2.5 \mu \mathrm{g} / \mathrm{mL}, 25 \mu \mathrm{g} / \mathrm{kg}$, i.p.) was obtained from Alomone Laboratories (Jerusalem, Israel), and Nissl stain was purchased from Beyotime Biotech (Jiangsu, China).

\section{Behavioral Assessments}

Neurobehavioral performance was evaluated at 1, 3, and 7 days after pMCAO using the Bederson score to evaluate the success of the stroke operation. ${ }^{6,14}$

\section{Horizontal Ladder Test}

The horizontal ladder test is used to measure the coordination of an animal walking on a horizontal ladder (width: $10 \mathrm{~cm}$, length: $107 \mathrm{~cm}$, height: $4 \mathrm{~cm}$ ) with wooden sticks located at $3-\mathrm{cm}$ intervals. The number of foot slides was recorded as a percentage ( $\%$ foot slides) by dividing the number of foot slides by the total number of runs. ${ }^{7}$

\section{5-Bromo-2'-Deoxyuridine (BrdU) Injections}

5-Bromo-2'-deoxyuridine (BrdU; Sigma, St. Louis, MO, USA) was used to examine the effects of catalpol on neurogenesis in rats subjected to stroke. Dosing with BrdU was performed according to previously described methods. ${ }^{15,16}$

\section{Tissue Preparation}

Tissues were prepared as described previously. ${ }^{9,11}$ Briefly, 7 days after stroke induction, rat brains were post-fixed in $4 \%$ formalin solution and sectioned into $10-\mu \mathrm{m}$ coronal sections using a cryostat (Leica CM1950, Oskar-Barnack, Germany) for immunohistochemical analysis. The ipsilateral ischemic cortex ( $0.1 \mathrm{~g}$ per brain) in each group was weighed in preparation for Western blot analysis. ${ }^{9,11}$

\section{Immunohistochemistry}

Immunofluorescent staining was performed on six separate coronal sections $(10 \mu \mathrm{m})$ as previously described. ${ }^{7,16}$ Mouse and rabbit anti-tubulin III (TuJ-1, 1:100) were purchased from Cell Signaling Technology (Danvers, MA, USA). Mouse anti-BrdU (1:300), rabbit anti-Nestin (1:50), cleaved caspase-3 (1:50), and Cy3-conjugated goat anti-mouse $\operatorname{IgG}(1: 100)$ were purchased from Proteintech (Wuhan, China). Fluorescein isothiocyanate (FITC)-conjugated goat anti-rabbit IgG (1:100) was purchased from ZSGB Biotech. Co. (Peking China). Immunostained cells were visualized using a Nikon microscope, and images were captured using a Nikon digital camera (Tokyo, Japan). Results of immunofluorescence staining for Nestin, TuJ-1 (green), and BrdU (red) were visualized using a confocal microscope (DFC310 FX, Leica, Oskar-Barnack, Germany). According to our previous studies, ${ }^{9-12}$ five fields from 
each slice were randomly selected for blinded scoring and analyses, and the number of double-stained cells and the staining intensity at the ischemic boundary zone were analyzed using Image Pro Plus Version 6.0 software (Media Cybernetics, Rockville, USA). Each experiment was performed in triplicate.

\section{Western Blotting}

Western blotting and cellular fractionation were performed as previously described. ${ }^{13,21}$ Brain tissues from three animals per group were evaluated for Western blotting, and each rat brain was analyzed in triplicate. The following antibodies were used at the indicated concentrations: mouse anti-tubulin III (TuJ-1, 1:200, Cell Signaling Technology, Inc, China); Bcl-2 (1:300, Bioss, Beijing, China); Bax (1:2000); BDNF (1:300); and TrkB (1:300) (Proteintech); rabbit anti-GAPDH (1:3000; Bioworld Technology, Louis Park, USA); horseradish peroxidase (GenScript, 1:5000, Nanjing, China); and anti-mouse horseradish peroxidase (GenScript, 1:5000). Immunoreactive bands were digitally scanned using a Tanon MP system and quantified using a Tanon Gis gel imaging system (Tanon, Shanghai, China). All Western blot bands were normalized to GAPDH as the internal control.

\section{Statistical Analyses}

Data were expressed as the mean \pm SD. All data were analyzed using a one-way analysis of variance (ANOVA) using SPSS 16.0 software (IBM, USA). $P<0.05$ was considered statistically significant.

\section{Results}

\section{Catalpol Improved Performance On The Horizontal Ladder Test}

After stroke, the number of left (impaired side) foot missteps was significantly higher than that in the sham group $(p<0.05)$, indicating that stroke affected the brain cortex. At 1 and 3 days after stroke, no significant differences were observed between the catalpol and model groups. In contrast, at 7 days post-stroke, the percentage of foot slips in the catalpol groups ( 5 and $10 \mathrm{mg} / \mathrm{kg}$ ) was significantly lower than that in the model group $(p<0.05)$. Spontaneous recovery of neurological function was also observed at 1 , 3 , and 7 days after focal ischemia, as evidenced by a gradual decrease in foot slips over time (Table 1).
Table I Percentage Of Foot Slips In The Horizontal Ladder Test At Different Time Points After Stroke $(\mathrm{N}=6)$

\begin{tabular}{|l|l|l|l|}
\hline Group & I Day & 3 Days & 7 Days \\
\hline Sham & $6.56 \pm 2.21 \%$ & $5.12 \pm 1.89 \%$ & $2.56 \pm 2.22 \%$ \\
\hline Model & $40.24 \pm 4.46 \% * *$ & $39.35 \pm 7.03 \% * *$ & $35.69 \pm 3.15 \% * *$ \\
\hline $\begin{array}{l}\text { Catalpol } \\
(5 \mathrm{mg} / \mathrm{kg})\end{array}$ & $38.13 \pm 7.26 \%$ & $37.55 \pm 7.89 \%$ & $27.95 \pm 5.67 \%^{\# \#}$ \\
\hline $\begin{array}{l}\text { Catalpol } \\
(10 \mathrm{mg} / \mathrm{kg})\end{array}$ & $40.46 \pm 4.61 \%$ & $38.86 \pm 4.20 \%$ & $29.02 \pm 4.41 \%^{\#}$ \\
\hline
\end{tabular}

Notes: $* * p<0.01$ vs. sham; ${ }^{\#} p<0.01,{ }^{\#} p<0.05$ vs. model.

\section{Catalpol Significantly Increased The Number Of Cells That Co-Expressed Nestin And BrdU In The Ischemic Boundary Of The Ipsilateral Rat Cortex}

Nestin is a marker of neuronal progenitor cells. ${ }^{17}$ Nerve stem cells are characterized by a small cytoplasm-to-cell body ratio, a large ratio of nuclei-to- cell body, deep dyeing, small diameter cells, and BrdU- and Nestin-positive staining. As shown in Figure 1, catalpol (5 and $10 \mathrm{mg} / \mathrm{kg}$ ) significantly increased the number of Nestin- and BrdUimmunoreactive cells in the ipsilateral brain cortex (Figure $1 \mathrm{~A}, p<0.01$ vs. model). Furthermore, catalpol also enhanced Nestin and BrdU co-expression in the ischemic boundary of the cortex (Figure 1A, $p<0.01$ vs. model). The mean density (Figure 1C) of Tuj-1- and BrdU-positive cells in the cortex in the infarction boundary was consistent with the immunostaining results (Figure 1B).

\section{Catalpol Significantly Increased The Number Of Cells That Co-Expressed Tuj- I And BrdU In The Ischemic Boundary Of The Ipsilateral Rat Cortex}

Newborn immature neurons were marked by double-labeling for BrdU and Tuj-1 (Figure 2A). Seven days after induction of stroke, BrdU/Tuj-1 double-positive newborn neurons were observed in the ischemic boundary of the cortex, whereas no new immature neurons were observed in the sham operation group. Tuj-1 staining occurs in the cytoplasm, and BrdU stains the nucleus. The cells shown in Figure 1 that expressed BrdU and Nestin were more primitive, which indicated that they were neural stem cells. Interestingly, treatment with catalpol (5 and $10 \mathrm{mg} / \mathrm{kg}$ ) resulted in increased BrdU/Tuj-1 double-positive cells 7 days after stroke ( $p<0.05$ vs. model). However, catalpol 


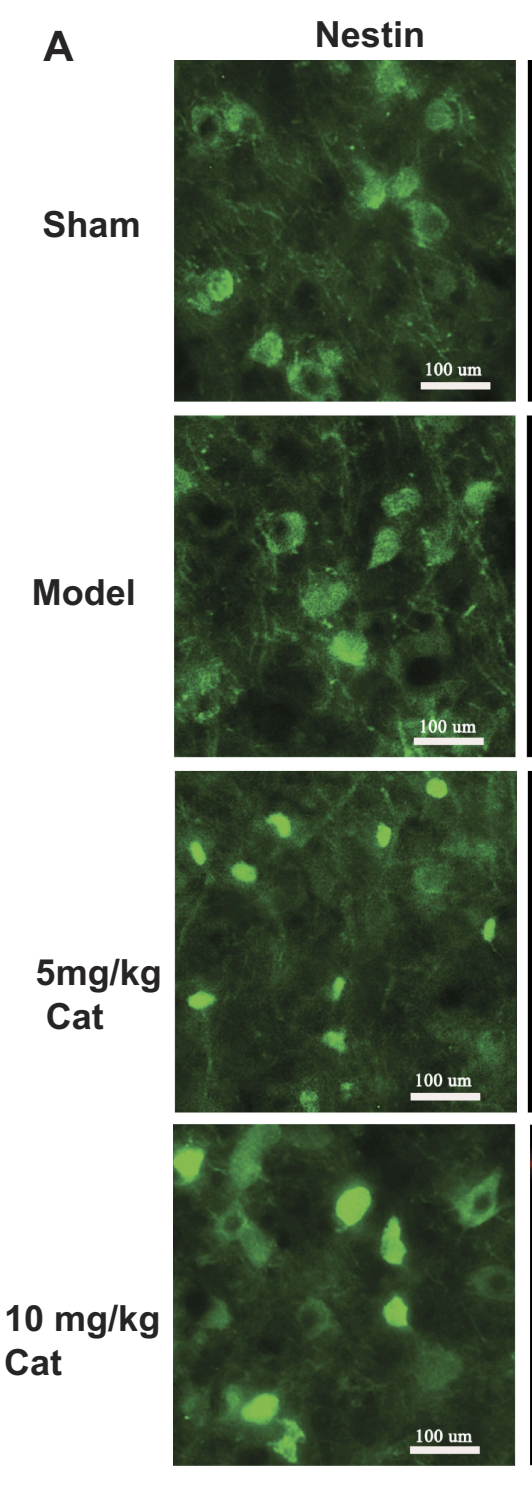

B

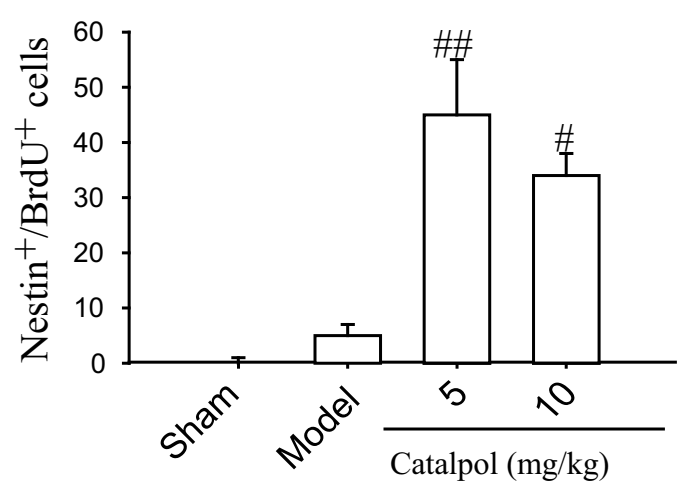

Brdu
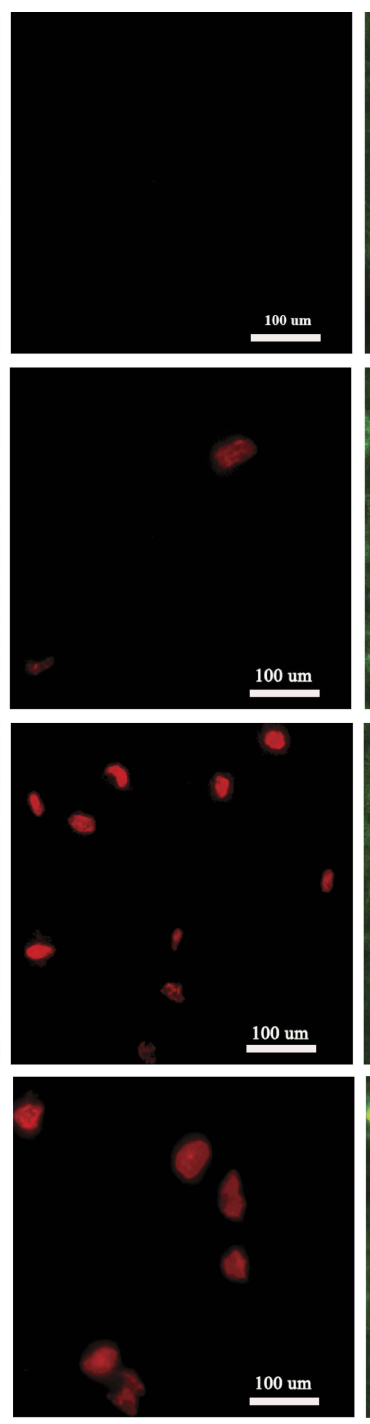

C
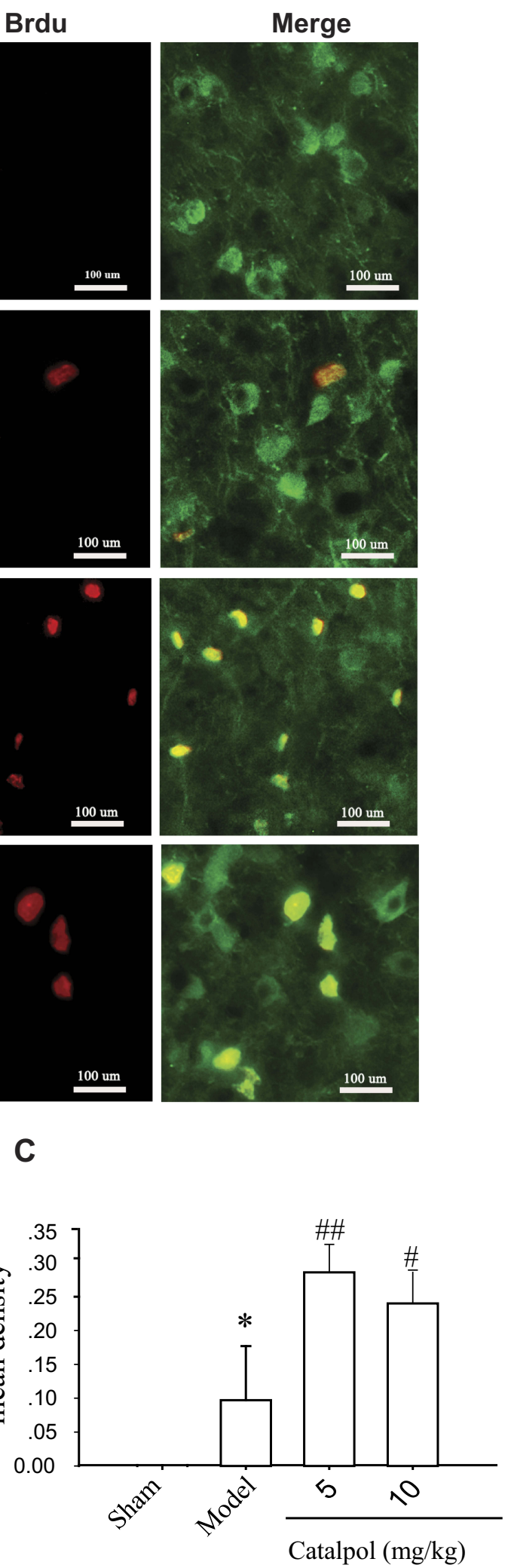

Figure I Catalpol promoted neurogenesis in the ischemic boundary of the ipsilateral rat cortex. (A) The effects of catalpol on neurogenesis were indicated by doublestaining for Nestin, a marker of neuronal progenitor cells, and BrdU, a marker of cell proliferation. Co-labeling of Nestin (green) and BrdU (red) demonstrated neurogenesis in the cortex ipsilateral to PMCAO after 7 days in each group. Co-localization of Nestin and BrdU in yellow is shown by the arrow $(400 \times$, bar $=100 \mu \mathrm{m})$. The number $(\mathbf{B})$ and mean density $(\mathbf{C})$ of cells co-labeled with Nestin and BrdU. ${ }^{*} p<0.05$ vs. sham, ${ }^{\#} p<0.05$ vs. model, ${ }^{\#} p<0.01$ vs model. 


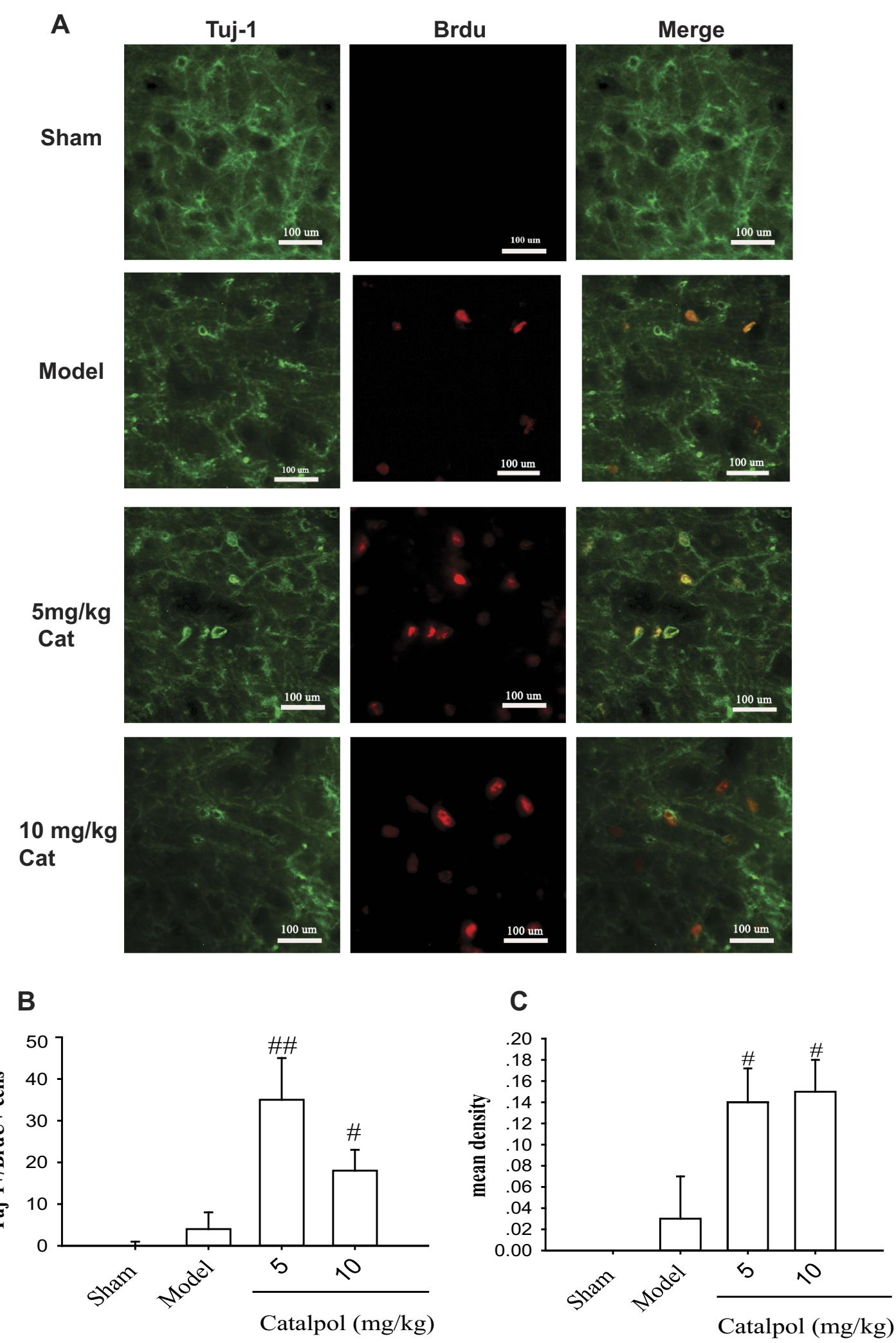

Figure 2 Catalpol promoted neural stem cell differentiation into newborn immature neurons. Newborn immature neurons were double-labeled for Tuj-I (green) and BrdU (red). (A) Tuj-I and BrdU immunostaining in the cortex of rat brain around the infarction ipsilateral to pMCAO after 7 days in each group. Co-localization of Tuj-I and BrdU in yellow is shown by the arrow $(400 \times$, bar $=100 \mu \mathrm{m})$. Number $(\mathbf{B})$ and mean density $(\mathbf{C})$ of Tuj-I- and BrdU-positive cells in the cortex around the infarction ipsilateral to PMCAO after 7 days in each group. ${ }^{\#} p<0.01$ vs model, ${ }^{\#} p<0.05$ vs model. 
increased double-positive BrdU/Tuj-1 staining regardless of dose (Figure 2A and B). The mean density results (Figure 2C) for $\mathrm{Tuj}_{-1}^{+}$and $\mathrm{BrdU}^{+}$cells in the cortex surrounding the infarction were consistent with the analysis of the number of co-stained cells (Figure 2B).

\section{Catalpol Downregulated Cleaved Caspase 3, As Assessed By Double- Fluorescent Staining In Vivo}

To determine whether catalpol reduced apoptosis of new neurons, sections from rats euthanized 7 days post-stroke were immunostained for cleaved caspase 3 (green) and Tuj1 (red) (Figure 3A). Catalpol increased the number of Tuj1+ cells (Figure 3B) and decreased the number of cleaved caspase $3+$ cells (Figure $3 \mathrm{C})(p<0.05$ vs. model). In addition, cells positive for both cleaved caspase 3 and Tuj 1 were significantly decreased in the $5 \mathrm{mg} / \mathrm{kg}$ catalpol group ( $p<0.05$ vs. model), while the decrease in doublestained cells in response to $10 \mathrm{mg} / \mathrm{kg}$ catalpol was not statistically significant (Figure 3D). Survival of Tuj $1^{+}$ cells was increased in the 5 and $10 \mathrm{mg} / \mathrm{kg}$ catalpol groups $(p<0.05$ vs model; Figure 3E). These results suggested that catalpol decreased the apoptosis of newborn neurons in the ischemic cortex of adult rat brains 7 days after pMCAO.

\section{Catalpol Enhanced Neurogenesis And New Neuron Survival Via BDNF, But Not Through The BDNF/TrkB Pathway}

We evaluated the effects of catalpol on the expression of BDNF and its receptor by Western blot analysis of BDNF, TrkB, and $\mathrm{p}-\mathrm{TrkB}$. The results showed that stroke reduced the expression of BDNF $(0.89 \pm 0.05)$. In contrast, $5 \mathrm{mg} /$ $\mathrm{kg}$ and $10 \mathrm{mg} / \mathrm{kg}$ catalpol $(1.02 \pm 0.05$ and $1.04 \pm 0.05 \mathrm{vs}$. model, $p<0.01)$ significantly increased the expression of BDNF (Figure 4A and B). However, the ratio of p-TrkB/ TrkB did not differ significantly among any of the groups post-stroke (Figure 4C and D).

We used K252a, a TrkB phosphorylase inhibitor, ${ }^{18}$ to further characterize the effects of catalpol on the expression of BDNF, TrkB, p-TrkB, Bcl-2, Bax, Tuj-1, and Map-2. The results showed that $\mathrm{K} 252 \mathrm{a}$ reduced the expression of $\mathrm{BDNF}$ (Figure 5A and B), and catalpol reversed the K252ainduced decrease in BDNF (0.95 \pm 0.06 vs. $0.60 \pm 0.10$, $p<0.01$, Figure $5 \mathrm{~A}$ and B). However, K252a did not affect the ratio of $\mathrm{p}-\mathrm{TrkB} / \mathrm{TrkB}$ in any group post-stroke (Figure $5 \mathrm{C}$ and D). Co-treatment with $\mathrm{K} 252 \mathrm{a}$ and $5 \mathrm{mg} / \mathrm{kg}$ catalpol increased the expression of Bcl-2 (Figure 5E and
F); Tuj-1 (Figure 5G and H); and MAP-2 (Figure 5I and J), and reduced the expression of $\mathrm{Bax} p<0.01$, compared to the model group (Figure 5E and F). There were no significant differences in the expression of any of these markers among the catalpol ( 5 and $10 \mathrm{mg} / \mathrm{kg}$ ), K252a, and K252a with 5 $\mathrm{mg} / \mathrm{kg}$ catalpol, groups. These results showed that catalpol enhanced neurogenesis and inhibited apoptosis of newborn neurons and that these effects may be related to BDNF, but not BDNF/TrkB, signaling (Figure 5).

\section{Catalpol And K252a Promoted Neuronal} Survival (Nissl staining)

Nissl bodies are large granular bodies found in neurons. Nissl bodies are indicators of protein synthesis in the cytoplasm and are seen as blue staining (Figure 6A). Following stroke, neuronal damage in the ipsilateral ischemic cortex was indicated by reduced Nissl body staining in the cytoplasm, and vacuolization (Figure 6B). Treatment with k252a resulted in similar histological findings as those in the model group (Figure 6C). In contrast, catalpol reduced neuronal damage, as evidenced by the increased number of Nissl bodies, more compact neuronal cell bodies, more pronounced staining, and more organized cellular arrangement than in the model group (Figure 6D-F). Catalpol ( $5 \mathrm{mg} / \mathrm{kg}$ ) only exerted stronger effects, as evidenced by more clearly visible neurites in the catalpol group compared with the model group (Figure 6E). The mean density (Figure 6G) results of Nissl body staining in each group were consistent with the tissue results reported in Figure 6A-F.

\section{Discussion}

Previous studies have shown that catalpol improved angiogenesis and neurological function in a rat stroke model. ${ }^{9,11}$ In this study, to our best knowledge, we showed for the first time that catalpol promoted neurogenesis at day 7 after stroke, and improved recovery of neural function (Figures 1, 2 and Table 1). One study showed that catalpol with puerarin (C-P) promoted angiogenesis and neurogenesis. ${ }^{12}$ Based on our previous studies, ${ }^{9,11}$ the effect of C-P on angiogenesis was partially due to the effects of catalpol, but the effects of catalpol on neurogenesis have not been previously characterized. In this study, catalpol promoted cell proliferation in the cortex surrounding the focal stroke site, and increased coexpression of $(\mathrm{BrdU})$ and Nestin ${ }^{17,19}$ in the ventricles and cortex, respectively (Figures 1 and 2). Moreover, the $\mathrm{BrdU}^{+} /$ Tuj $1^{+}$cell count was increased in the ischemic boundary regions following catalpol treatment (Figure 2A and B), 
A

DAPI

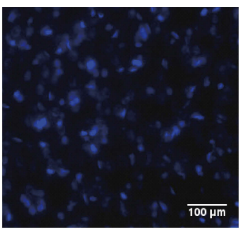

Sham

Model
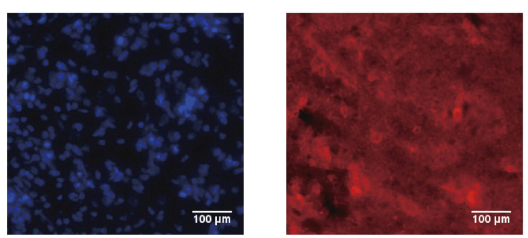

Catalpol

$5 \mathrm{mg} / \mathrm{kg}$
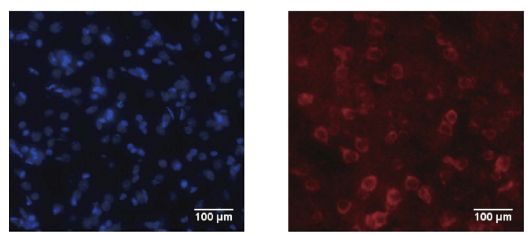

Catalpol

$10 \mathrm{mg} / \mathrm{kg}$
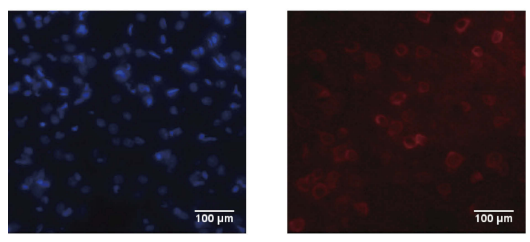

B

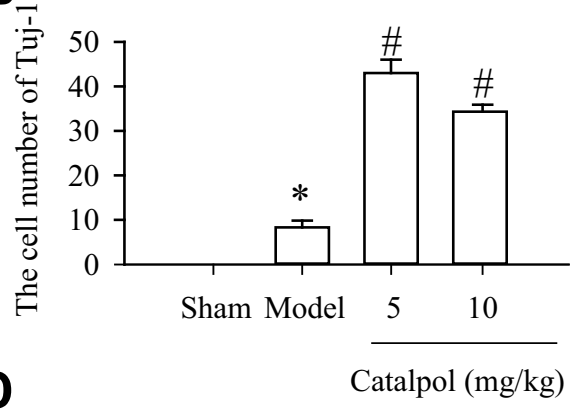

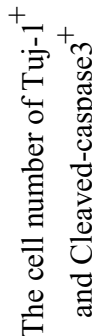

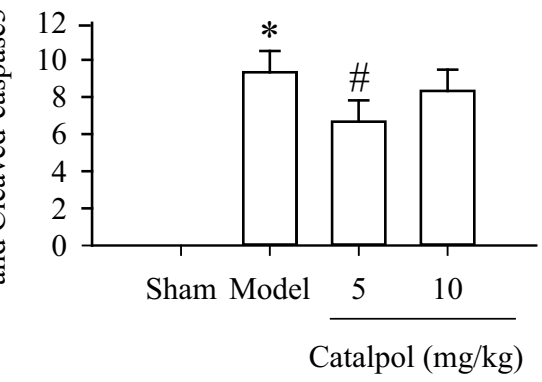

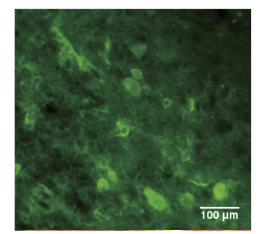
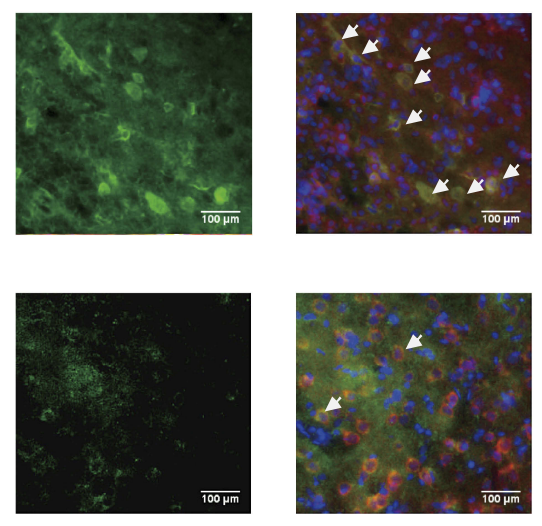

Cleaved-
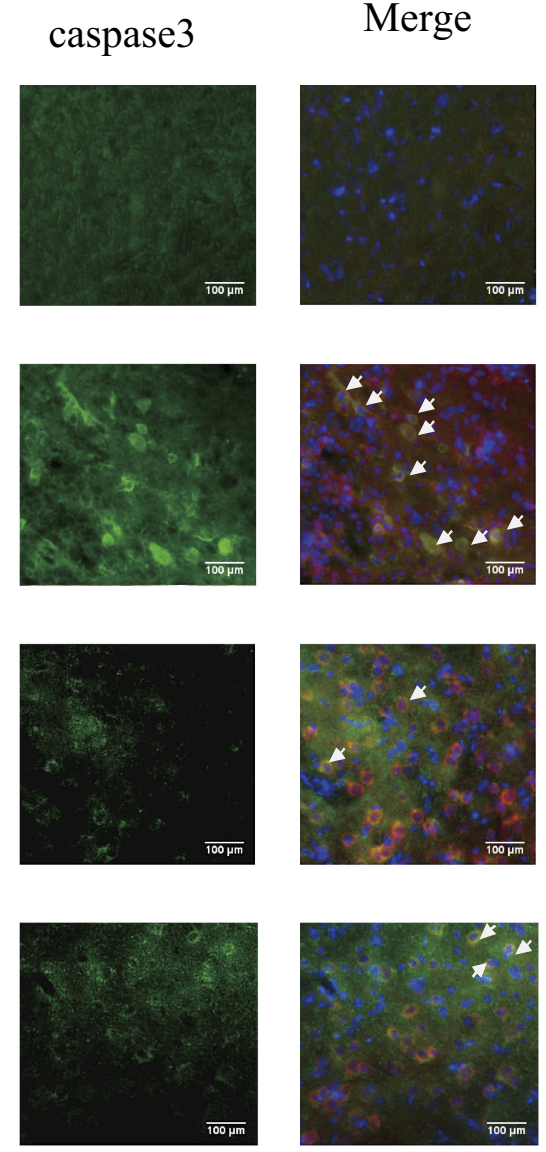

C

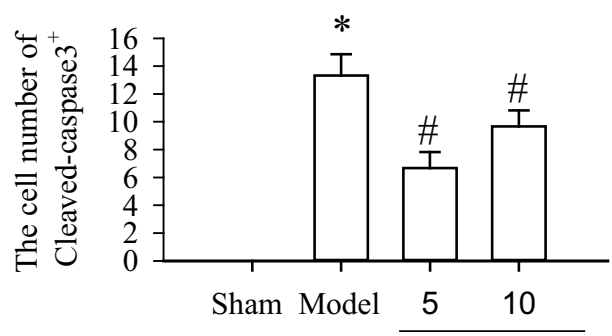

E

Catalpol (mg/kg)

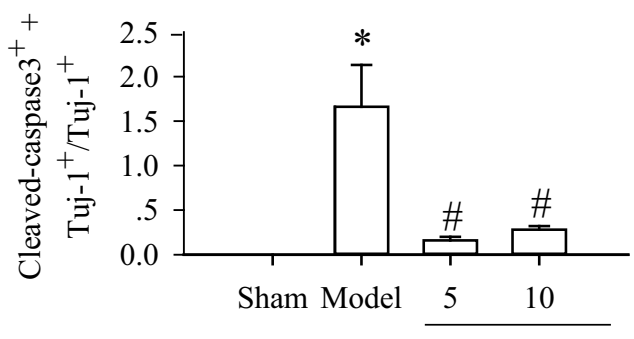

Catalpol (mg/kg)

Figure 3 Catalpol promoted survival newborn neurons via downregulating Cleaved-caspase-3. (A) The effects of catalpol on the survival of newborn neurons were indicated by double-staining for Tuj- I (cy3, red), a marker of newborn neurons. and for cleaved caspase 3 (alexa-488, green), a marker of cell apoptosis $(200 \times$, bar $=100 \mu \mathrm{m})$. Co-labeling of apoptosis (green) and Tuj-I (red) showed apoptotic newborn neurons in the peri-infarcted area at 7 days after pMCAO. Co-localization of Tuj-I and cleaved caspase 3 is shown in yellow. Statistical analyses are shown for the number of Tuj-I (B), cleaved caspase 3 (C), co-Tuj-I and cleaved caspase 3 (D), and the ratio of cells costained with cleaved caspase 3 and Tuj-I among Tuj-I-positive cells (E). ${ }^{*} p<0.05$ vs. sham, ${ }^{\#} p<0.05$ vs. model. 
A

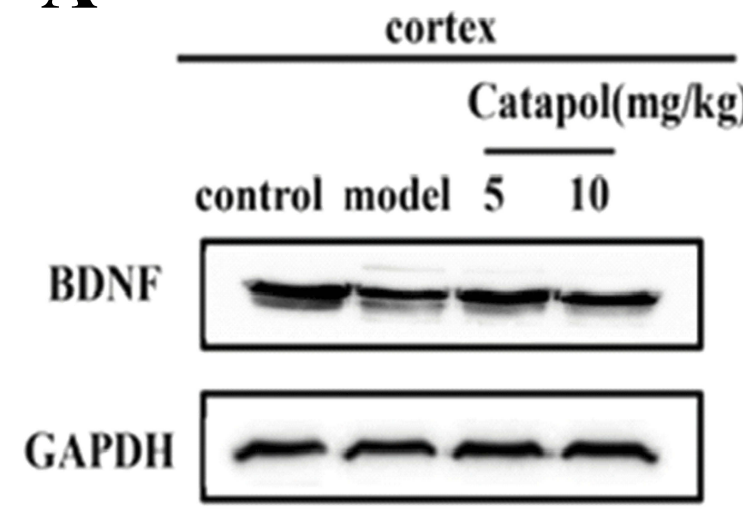

C

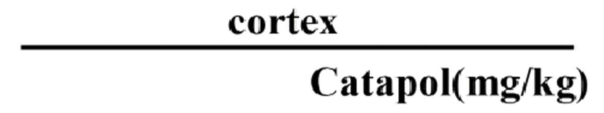

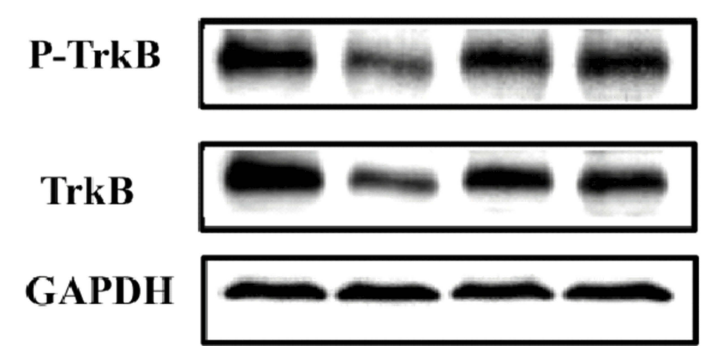

B

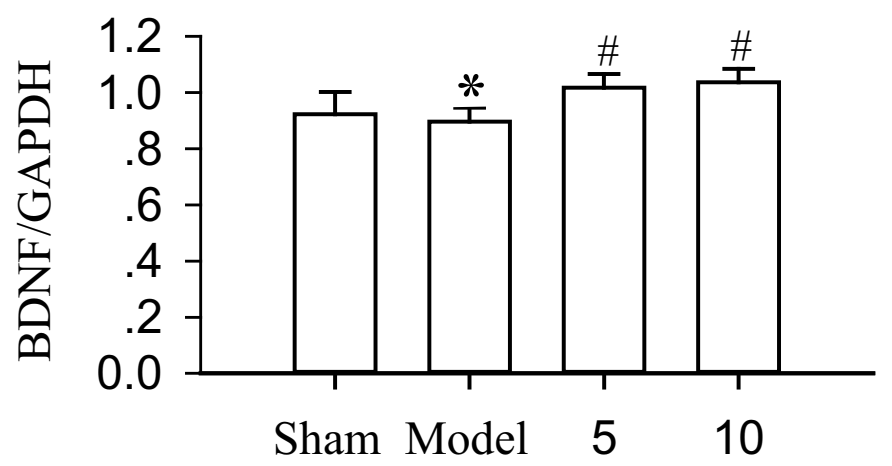

Catalpol (mg/kg)

D

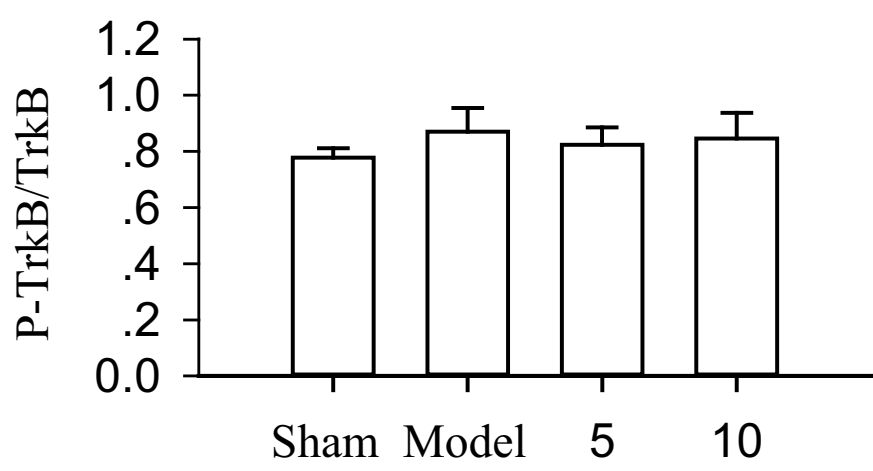

Catalpol (mg/kg)

Figure 4 Catalpol enhanced neurogenesis and survival of new neurons through BDNF. BDNF (A), P-TrkB, and TrkB (C) protein expression in the rat brain cortex was detected using Western blot. The internal control was GAPDH. The experiments were repeated three times, and 6 rats were used in each group. Statistical bars shown are shown in (B) and (D). ${ }^{*} p<0.05$ vs sham, ${ }^{\#} p<0.01$ vs model.

which indicated that some neuronal stem cells differentiated into neurons in response to catalpol treatment.

Approximately $\geq 80 \%$ of new neurons die during the first 6 weeks after stroke, with only about $0.2 \%$ of dead striatal neurons replaced during this period. ${ }^{13,20}$ Therapeutic strategies should focus on enhancing the survival of newborn neurons, which may later incorporate into circuits with existing neurons in-situ. ${ }^{21}$ In our study, catalpol enhanced neurogenesis in the SVZ (data not shown) and the ischemic boundary regions of the cortex, as evidenced by decreased co-labeling of Tuj 1 and cleaved caspase 3 following stroke. Although co-expression of cleaved caspase 3 and Tuj1 was significantly reduced only in the $5 \mathrm{mg} / \mathrm{kg}$ catalpol group, the survival of Tuj1-positive cells was increased in both the 5 and $10 \mathrm{mg} / \mathrm{kg}$ catalpol groups $(p<0.05$ vs. model) (Figure $3 \mathrm{~A}-\mathrm{E})$. These results suggested that catalpol reduced apoptosis of newborn neurons following stroke, resulting in enhanced survival.

The immunostained pattern of Tuj-1 between Figures 2 and 3. Looks like different. In fact, the form is the same and there are some differences in the background. To observe whether the Tuj-1 positive cell experienced apoptosis. Tuj-1 in Figure 3 Stained in the cytoplasm, and increased staining indicates more mature neurons. Nuclear BrdU with Nestin staining in Figure 1 indicates stem cells differentiating into neurons, which is almost different from the previous Figure 1, BrdU and Nestin representing proliferating nuclei, the nuclei/cell body near to 1, indicating the BrdU and Nestin both positive cells shown in Figure 1 were more primitive, 
A

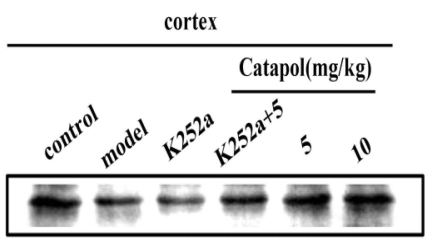

GAPDH
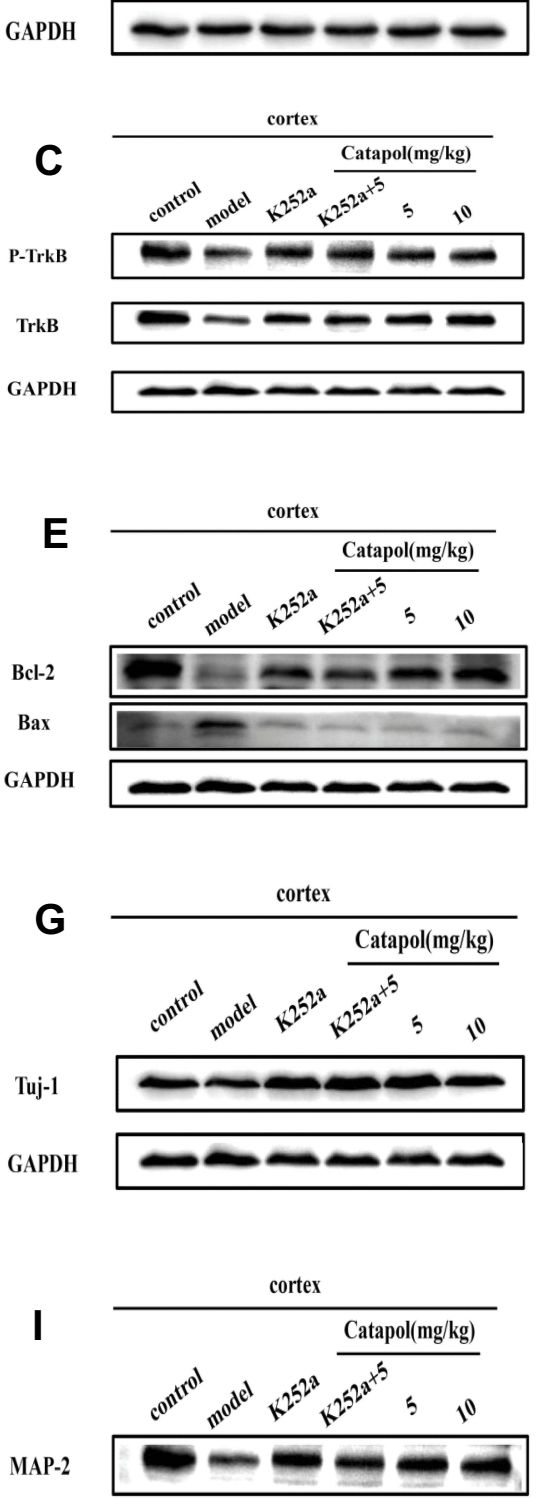

GAPDH
B

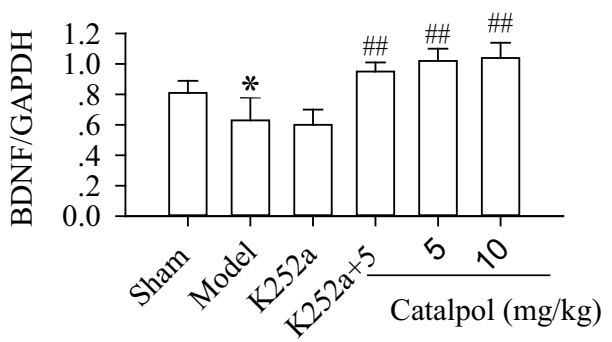

D
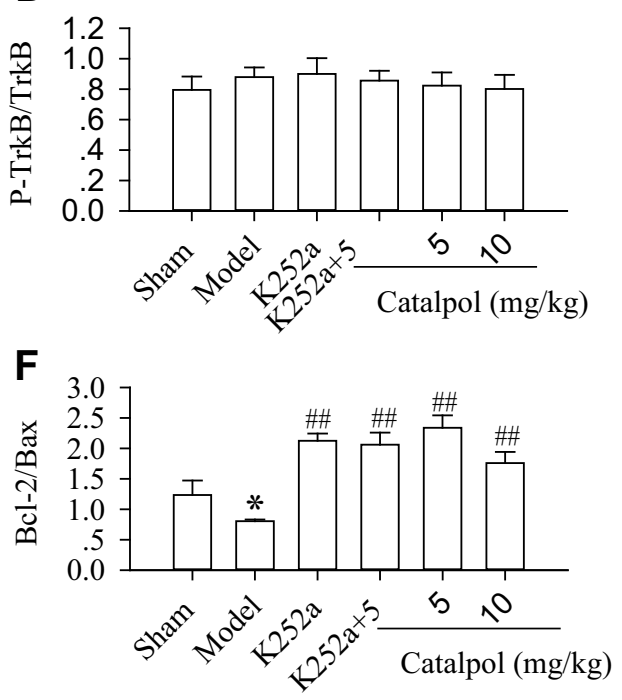

H

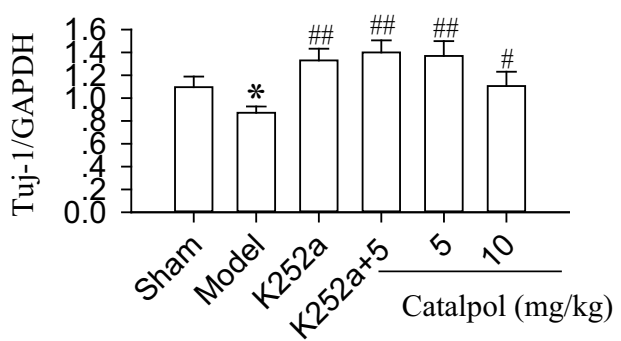

J

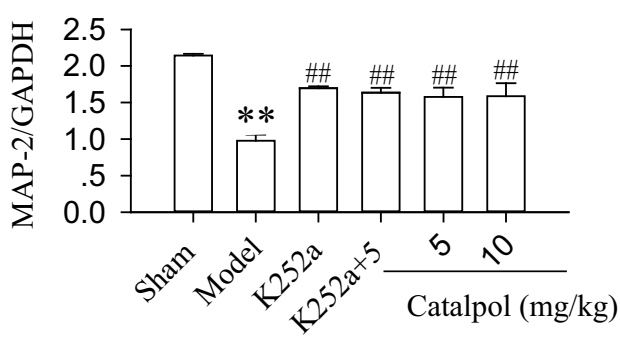

Figure 5 Enhanced neurogenesis and new neuron survival by catalpol were related to BDNF, but not the BDNF/TrkB pathway. Immunoblot analyses of BDNF (A), TrkB/P$\operatorname{TrkB}(\mathbf{C}), \mathrm{Bcl}-2 / \mathrm{Bax}(\mathbf{E})$, Tuj-I (G), and Map-2 (I) protein in the rat brain cortex surrounding the ischemic core 7 days after ischemia. Statistical bars are shown in (B), (D), $(\mathbf{F}),(\mathbf{H})$, and $(\mathbf{J}) .{ }^{*} p<0.05$ and ${ }^{* *} p<0.01$ vs sham, ${ }^{*} p<0.05$ vs model, ${ }^{\#} p<0.01$ vs model. The internal control was GAPDH. The experiments were repeated three times and 6 rats were used in each group. 


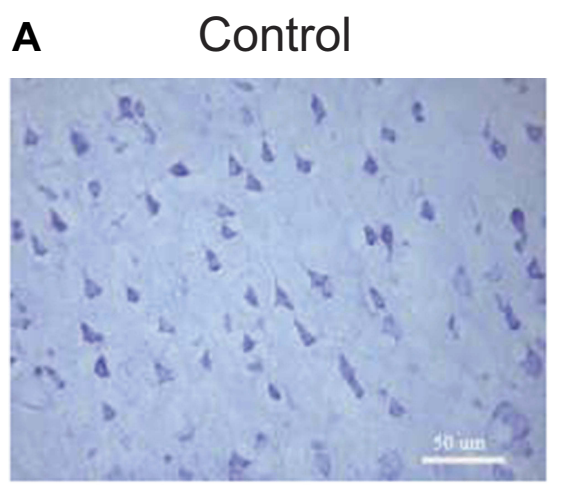

D
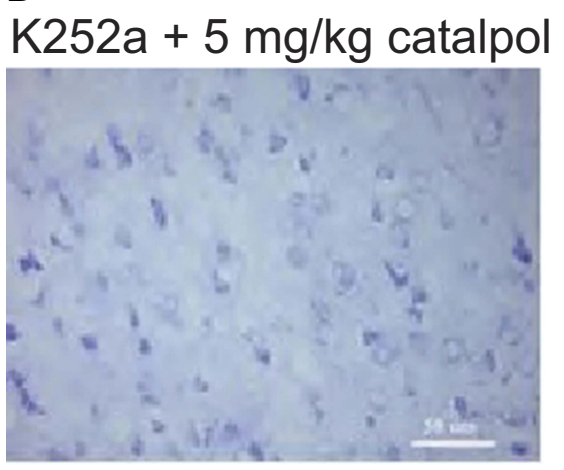

B

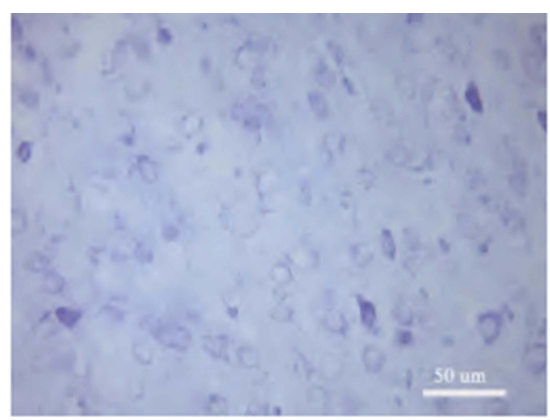

E

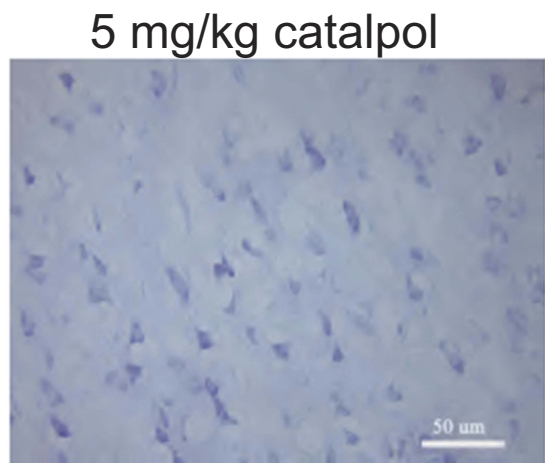

C

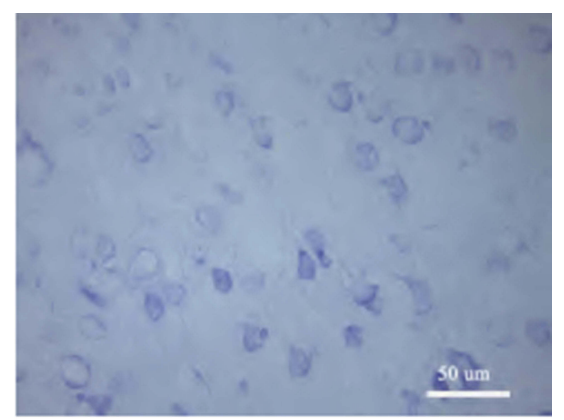

$\mathbf{F}$

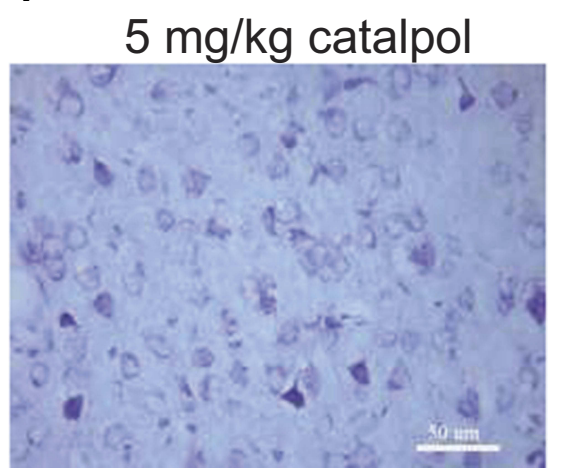

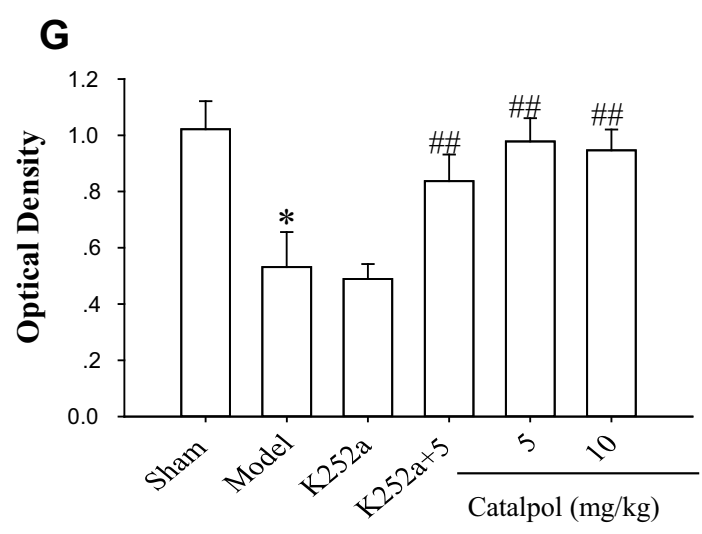

Figure 6 Catalpol promoted neuronal survival, as evidenced by Nissl staining. Nissl staining showed more neuronal damage in the ipsilateral ischemic cortex, whereas catalpol, and K252a with catalpol, improved neuronal damage 7 days after stroke (400x, bar = $50 \mu \mathrm{m})$. (A) Control; (B) Model; (C) K252a; (D) K252a + 5 mg/kg catalpol; (E) $5 \mathrm{mg} / \mathrm{kg}$ catalpol; (F) $10 \mathrm{mg} / \mathrm{kg}$ catalpol; (G) Optical density of each group. ${ }^{*} \mathrm{p}<0.05$ and \#\# $<0.01$ vs model.

which indicated that they were neural stem cells. Previous studies showed that only a small population of newly generated neurons survives after stroke. ${ }^{13,20-22} \mathrm{Bcl}-2$, an inhibitor of apoptosis, and Bax, a promotor of apoptosis, regulate the survival of new neurons. ${ }^{23,24}$ We showed that stroke induced Bax expression and decreased Bcl-2 expression, and catalpol reversed this effect (Figures 4 and 5). In addition, catalpol induced recovery of neurobehavioral function, increased Nissl staining (Figure 6), enhanced neurogenesis, and prevented the death of new neurons by inhibiting apoptosis.
Various neurotrophic and growth factors, such as BDNF, EPO, and VEGF, can stimulate neurogenesis and inhibit apoptosis. ${ }^{4-8}$ Catalpol has been shown to promote BDNF expression and increase EPO and VEGF secretion, ${ }^{9-11}$ which may provide a favorable environment for new neurons to survive and integrate with existing tissue. Moreover, the pro-angiogenic effects of catalpol may restructure the microcirculation, resulting in the delivery of oxygen and nutrients to newborn neurons. ${ }^{9-11,24,25}$ 
Mature neurons may derive from neurogenesis in the SVZ, the ischemic area, or from neurons that survived the ischemic event. ${ }^{1,2,27,28}$ The number of mature neurons increased in the stroke core following catalpol treatment in the cortex surrounding the stroke core, as shown by increased Map2, a marker of mature neurons (Figure 5). Catalpol significantly increased the expression of Tuj-1 protein (a newborn neuronal marker) and Map2 protein (Figures 1, 2, 5, and 6). Although spontaneous recovery after focal ischemia was observed within 1-7 days after stroke, catalpol further improved functional outcomes following stroke, as evidenced by decreased foot slips compared to the model group (Figure 1 and Table 1). These results indicated that catalpol affected neural functional outcomes following stroke through neurogenesis.

To evaluate the role of BDNF/TrkB signaling in the survival of nascent neurons following catalpol treatment, a membrane-permeable Trk tyrosine kinase inhibitor, K252a, was used. ${ }^{18}$ In the present study, catalpol promoted increased expression of BDNF, but not TrkB, which suggested that catalpol promoted neurogenesis and neuronal survival via mechanisms unrelated to the BDNF/TrkB signaling pathway. However, K252a treatment did not alter the $\mathrm{p}-\mathrm{TrkB} / \mathrm{TrkB}$ ratio, nor did catalpol $(5$ or $10 \mathrm{mg} /$ $\mathrm{kg}$ ), K252a alone, or K252a with $5 \mathrm{mg} / \mathrm{kg}$ catalpol. In contrast, treatment with catalpol ( 5 or $10 \mathrm{mg} / \mathrm{kg}), \mathrm{K} 252 \mathrm{a}$ alone, or $\mathrm{K} 252 \mathrm{a}$ with $5 \mathrm{mg} / \mathrm{kg}$ catalpol resulted in increased expression of Bcl-2, Tuj-1, and Map-2, and reduced expression of Bax, compared with the model group (Figure 5). Consistent with our results, recent studies showed that administration of BDNF into the lateral ventricle or to the SVZ stimulated neurogenesis. While TrkB was not essential for adult SVZ neurogenesis, the role of BDNF/TrkB in neurogenesis remains unclear. ${ }^{26,29}$ These results suggested that BDNF may promote neurogenesis and reduce apoptosis to promote new neuron survival via TrkB-independent mechanisms. Our results showed that catalpol enhanced neurogenesis and inhibited apoptosis of newborn neurons through BDNF-related mechanisms independent of TrkB signaling. Nissl bodies change under various physiological conditions and in pathological conditions and may dissolve and disappear (chromatolysis). As such, we used Nissl staining to evaluate the neurohistology of the brain. As shown in Figure 6, Nissl granules were distributed in the cytoplasm and dendrites of neurons, and deep staining was clearly observed in the control group. In contrast, neurons subjected to stroke were aberrant in shape, faintly stained, exhibited degeneration, and exhibited unclear cell contours. Treatment with $5 \mathrm{mg} / \mathrm{kg}$ catalpol reversed these effects, and also enhanced the effects of K252a, as evidenced by increased Nissl bodies and increased staining density. The effects of K252a in our study agreed with previous reports that K252a suppressed neuronal apoptosis through inhibition of MLK3/JNK-induced translocation of Bax to the mitochondrial membrane following transient global brain ischemia. $^{29}$

\section{Conclusions}

Our study showed that catalpol restored adult neurogenesis and upregulated BDNF following stroke. Catalpol induced neurogenesis at 7 days after stroke. Future studies should evaluate longer time courses of catalpol-induced recovery. Moreover, experiments using specific inhibitors or other methods such as siRNA or gene knockout should be performed to evaluate the relationship between the neurogenesis and Trk signaling.

\section{Abbreviations}

pMCAO, permanent middle cerebral artery occlusion; BDNF, brain-derived neurotrophic factor; EPO, erythropoietin; VEGF, vascular endothelial growth factor.

\section{Ethics Approval And Informed Consent}

All experiments were performed in accordance with the Basel Declaration and China's Guidelines for Care and Use of Laboratory Animals. The experimental protocol was approved by the Experimental Animal Ethics Committee of College of Pharmaceutical Sciences \& Chinese Medicine, Southwest University.

\section{Data Availability}

The datasets used and/or analyzed in the current study are available from the corresponding author upon reasonable request.

\section{Acknowledgment}

The authors gratefully acknowledge Allan V. Kalueff, Ph.D. for his review and helpful comments regarding this paper.

\section{Author Contributions}

All authors were involved in the design. Yali Shao, Lei Qin, and Jing-Huan Wang performed the study. Jing-Huan Wang, Yali Shao, Lei Qin, and Shan Feng performed 
acquisition, processing, analysis, and interpretation of data, and helped to revise the paper. Hui-Feng Zhu, Dong Wan, Jing-Huan Wang, and Yun-Bin Jiang wrote the manuscript. Hui-Feng Zhu and Dong Wan were principal investigators and corresponding authors. All authors gave final approval of the version to be published, and agree to be accountable for all aspects of the work.

\section{Funding}

This work was supported by grants from the National Natural Science Foundation of China (81873034), the Fundamental Research Funds for the Central Universities (XDJK201 2B010), and the Natural Science Foundation Project of CQ CSTC (cstc2014jcyjA10083; 2018jcyjAX0158).

\section{Disclosure}

The authors report no conflicts of interest in this work.

\section{References}

1. Zhang RL, Zhang ZG, Chopp M. Neurogenesis in the adult ischemic brain: generation, migration, survival, and restorative therapy. Neuroscientist. 2005;11(5):408-416. doi:10.1177/1073858405278865

2. Zhang R, Zhang Z, Wang L, et al. Activated neural stem cells contribute to stroke-induced neurogenesis and neuroblast migration toward the infarct boundary in adult rats. J Cereb Blood Flow Metab. 2004;24(4):441-448. doi:10.1097/00004647-200404000-00009

3. Goldman SA. Stem and progenitor cell-based therapy of the central nervous system: hopes, hype, and wishful thinking. Cell Stem Cell. 2016;18(2):174-188. doi:10.1016/j.stem.2016.01.012

4. Greenberg DA, Jin K. Growth factors and stroke. NeuroRx. 2006;3 (4):458-465. doi:10.1016/j.nurx.2006.08.003

5. Jessberger S. Neural repair in the adult brain. F1000Res. 2016;5. doi:10.12688/f1000research.7459.1

6. Komitova M, Mattsson B, Johansson BB, Eriksson PS. Enriched environment increases neural stem/progenitor cell proliferation and neurogenesis in the subventricular zone of stroke-lesioned adult rats. Stroke. 2005;36(6):1278-1282. doi:10.1161/01.STR.0000166197.94147.59

7. Li Y, Lu Z, Keogh CL, Yu SP, Wei L. Erythropoietin-induced neurovascular protection, angiogenesis, and cerebral blood flow restoration after focal ischemia in mice. J Cereb Blood Flow Metab. 2007;27(5):1043-1054. doi:10.1038/sj.jcbfm.9600417

8. Sun Y, Jin K, Xie L, et al. VEGF-induced neuroprotection, neurogenesis, and angiogenesis after focal cerebral ischemia. J Clin Invest. 2003;111(12):1843-1851. doi:10.1172/JCI200317977

9. Dong W, Xian Y, Yuan W, et al. Catalpol stimulates VEGF production via the JAK2/STAT3 pathway to improve angiogenesis in rats' stroke model. J Ethnopharmacol. 2016;191:169-179. doi:10.1016/j. jep.2016.06.030

10. Wan D, Xue L, Zhu H, Luo Y. Catalpol induces neuroprotection and prevents memory dysfunction through the cholinergic system and BDNF. Evid Based Complement Alternat Med. 2013;2013:134852. doi:10.1155/2013/134852

11. Zhu HF, Wan D, Luo Y, Zhou JL, Chen L, Xu XY. Catalpol increases brain angiogenesis and up-regulates VEGF and EPO in the rat after permanent middle cerebral artery occlusion. Int J Biol Sci. 2010;6 (5):443-453. doi:10.7150/ijbs.6.443
12. Liu Y, Xue Q, Li X, et al. Amelioration of stroke-induced neurological deficiency by lyophilized powder of catalpol and puerarin. Int J Biol Sci. 2014;10(4):448-456. doi:10.7150/ijbs.8571

13. Arvidsson A, Collin T, Kirik D, Kokaia Z, Lindvall O. Neuronal replacement from endogenous precursors in the adult brain after stroke. Nat Med. 2002;8(9):963-970. doi:10.1038/nm747

14. Bederson JB, Pitts LH, Tsuji M, Nishimura MC, Davis RL, Bartkowski H. Rat middle cerebral artery occlusion: evaluation of the model and development of a neurologic examination. Stroke. 1986;17(3):472-476. doi:10.1161/01.STR.17.3.472

15. Kawai T, Takagi N, Miyake-Takagi K, Okuyama N, Mochizuki N, Takeo S. Characterization of BrdU-positive neurons induced by transient global ischemia in adult hippocampus. J Cereb Blood Flow Metab. 2004;24(5):548-555. doi:10.1097/00004647-20040500000009

16. Kim JY, Choi K, Shaker MR, et al. Promotion of cortical neurogenesis from the neural stem cells in the adult mouse subcallosal zone. Stem Cells. 2016;34(4):888-901. doi:10.1002/stem.v34.4

17. Reynolds BA, Weiss S. Generation of neurons and astrocytes from isolated cells of the adult mammalian central nervous system. Science. 1992;255(5052):1707-1710. doi:10.1126/science.1553558

18. Cheng PL, Song AH, Wong YH, Wang S, Zhang X, Poo MM. Selfamplifying autocrine actions of BDNF in axon development. Proc Natl Acad Sci USA. 2011;108(45):18430-18435. doi:10.1073/ pnas. 1115907108

19. Sohur US, Emsley JG, Mitchell BD, Macklis JD. Adult neurogenesis and cellular brain repair with neural progenitors, precursors and stem cells. Philos Trans R Soc Lond B Biol Sci. 2006;361(1473):14771497. doi:10.1098/rstb.2006.1887

20. Lindvall O, Kokaia Z. Stem cell research in stroke: how far from the clinic? Stroke. 2011;42(8):2369-2375. doi:10.1161/STROKEAHA. 110.599654

21. Obernier K, Tong CK, Alvarez-Buylla A. Restricted nature of adult neural stem cells: re-evaluation of their potential for brain repair. Front Neurosci. 2014;8:162. doi:10.3389/fnins.2014.00162

22. Ming GL, Song H. Adult neurogenesis in the mammalian brain: significant answers and significant questions. Neuron. 2011;70 (4):687-702. doi:10.1016/j.neuron.2011.05.001

23. Sasaki T, Kitagawa K, Yagita Y, et al. Bcl2 enhances survival of newborn neurons in the normal and ischemic hippocampus. $J$ Neurosci Res. 2006;84(6):1187-1196. doi:10.1002/(ISSN)1097-4547

24. Liu J, Wang Y, Akamatsu Y, et al. Vascular remodeling after ischemic stroke: mechanisms and therapeutic potentials. Prog Neurobiol. 2014;115:138-156. doi:10.1016/j.pneurobio.2013.11.004

25. Xiong Y, Mahmood A, Chopp M. Angiogenesis, neurogenesis and brain recovery of function following injury. Curr Opin Investig Drugs. 2010;11(3):298-308.

26. Galvao RP, Garcia-Verdugo JM, Alvarez-Buylla A. Brain-derived neurotrophic factor signaling does not stimulate subventricular zone neurogenesis in adult mice and rats. J Neurosci. 2008;28(50):1336813383. doi:10.1523/JNEUROSCI.2918-08.2008

27. Zhang RL, Zhang ZG, Chopp M. Ischemic stroke and neurogenesis in the subventricular zone. Neuropharmacology. 2008;55(3):345352. doi:10.1016/j.neuropharm.2008.05.027

28. Ginsberg MD. Neuroprotection for ischemic stroke: past, present and future. Neuropharmacology. 2008;55(3):363-389. doi:10.1016/j. neuropharm.2007.12.007

29. Wang Q, Yin XH, Liu Y, GY Z. K252a suppresses neuronal cells apoptosis through inhibiting the translocation of Bax to mitochondria induced by the MLK3/JNK signaling after transient global brain ischemia in rat hippocampal CA1 subregion. J Recept Signal Transduct Res. 2011;31(4):307-313. doi:10.3109/10799893.2011.592536 


\section{Publish your work in this journal}

Drug Design, Development and Therapy is an international, peerreviewed open-access journal that spans the spectrum of drug design and development through to clinical applications. Clinical outcomes, patient safety, and programs for the development and effective, safe, and sustained use of medicines are a feature of the journal, which has also

been accepted for indexing on PubMed Central. The manuscript management system is completely online and includes a very quick and fair peer-review system, which is all easy to use. Visit http://www. dovepress.com/testimonials.php to read real quotes from published authors.

Submit your manuscript here: https://www.dovepress.com/drug-design-development-and-therapy-journal 